\title{
Nursing now Brasil: conhecimentos e perspectivas dos enfermeiros de uma unidade de pronto atendimento
}

RESUMO | Objetivo: Compreender os conhecimentos e perspectivas dos enfermeiros sobre a campanha Nursing Now Brasil. Método: Trata-se de uma pesquisa descritiva exploratória, com abordagem qualitativa, usando a metodologia "Bola de Neve". A técnica de "Bola de Neve" consiste em amostra não probabilística onde os indivíduos selecionados inicialmente indicam outros participantes. A População foi constituída por enfermeiros da Unidade de Pronto Atendimento de Sarandi/PR, as entrevistas foram gravadas e posteriormente transcritas e analisadas pela análise de conteúdo de Bardin. Resultados: Emergiram três categorias: Diferentes percepções e conhecimentos da campanha Nursing Now Brasil pelos enfermeiros, Desconhecimento dos enfermeiros sobre a campanha Nursing Now Brasil e Melhorias na enfermagem: esperança versus desvalorização. Conclusão: Evidenciou-se que a campanha NursingNow Brasil ainda é pouco conhecida e divulgada entre os profissionais de enfermagem, nota-se também a presença do sentimento de esperança na busca constante por melhorias e valorização da classe profissional.

Palavras-chaves: Organização Pan-Americana da Saúde; Enfermagem; Brasil.

ABSTRACT | Objective: To understand nurses' knowledge and perspectives on the Nursing Now Brazil campaign. Method: This is an exploratory descriptive research, with a qualitative approach, using the "Snowball" methodology. The "Snowball" technique consists of a non-probabilistic sample where the individuals selected initially indicate other participants. The population consisted of nurses from the Sarandi / PR Emergency Care Unit, the interviews were recorded and later transcribed and analyzed by Bardin's content analysis. Results: Three categories emerged: Different perceptions and knowledge of the Nursing Now Brazil campaign by nurses, Ignorance of nurses about the Nursing Now Brazil campaign and Improvements in nursing: hope versus devaluation. Conclusion: It became evident that the Nursing Now Brazil campaign is still little known and disseminated among nursing professionals, there is also the presence of the feeling of hope in the constant search for improvements and enhancement of the professional class.

Keywords: Pan American Health Organization; Community Health Nursing; Brazil.

RESUMEN | Objetivo: Comprender los conocimientos y perspectivas de las enfermeras sobre la campaña Nursing Now Brazil. Método: Se trata de una investigación descriptiva exploratoria, con enfoque cualitativo, utilizando la metodología "Snowball". La técnica "Snowball" consiste en una muestra no probabilística donde los individuos seleccionados inicialmente indican otros participantes. La población estuvo conformada por enfermeras de la Unidad de Emergencias de Sarandí / PR, las entrevistas fueron grabadas y luego transcritas y analizadas por el análisis de contenido de Bardin. Resultados: Surgieron tres categorías: Diferentes percepciones y conocimientos de las enfermeras sobre la campaña Nursing Now Brazil, Desconocimiento de las enfermeras sobre la campaña Nursing Now Brazil y Mejoras en enfermería: esperanza versus devaluación. Conclusión: Se hizo evidente que la campaña Nursing Now Brazil aún es poco conocida y difundida entre los profesionales de enfermería, también existe la presencia del sentimiento de esperanza en la búsqueda constante de mejoras y perfeccionamiento de la clase profesional.

Palavras claves: Organización Panamericana de La Salud; Enfermeríaen Salud Comunitaria.

\section{Vinícius Brito de Souza}

Acadêmico do curso de Enfermagem, Centro Universitário de Maringá (UNICESUMAR), Bolsista PIBIC-UniCesumar.

ORCID: 0000-0002-1236-5379

\section{Eloísa Lopes do Amaral}

Acadêmica do curso de Enfermagem, Centro Universitário de Maringá (UNICESUMAR).

ORCID: 0000-0002-9982-9913

\section{Geisa dos Santos Luz}

Enfermeira, Doutora, Docente do curso de Medicina do Centro Universitário de Maringá (UNICESUMAR).

ORCID: 0000-0002-3342-3320

\section{Marcos Benatti Antunes}

Enfermeiro, Doutor, Docente do curso de Medicina do Centro Universitário de Maringá (UNICESUMAR).

ORCID: 0000-0002-5139-8827
Recebido em: 24/08/2020

Aprovado em: 07/10/2020

INTRODUÇÃo

$\Lambda$ enfermagem é a arte de cuidar e constitui a metade do trabalho na área da saúde, além disso os enfermeiros assumem o papel de gestor e coordenador da equipe e em diversas áreas na saúde, em diferentes pontos de Atenção à Saúde ${ }^{(1)}$.

A Organização Pan-Americana da Saúde (OPAS) e Organização Mundial da Saúde (OMS), desde 2013, vem discutindo a ampliação do papel do enfermeiro, focando na atuação na Atenção Primária a Saúde (APS), no que tange a gestão e assistência dos cuidados ao paciente ${ }^{(2)}$.

Embora haja esse reconhecimento do papel do enfermeiro, há algumas limita- ções relacionadas a valorização da equipe de enfermagem, a exemplo, a carga horária exaustiva, número insuficiente de profissionais nas unidades de saúde, capacitações e formação permanente e ainda avanços na autonomia dos profissionais.

Nesse contexto, surge a campanha mundial de fortalecimento da enfermagem, denominada Nursing Now, que chega ao Brasil em 2019, com as seguintes metas para 2020:

[...] Maior investimento na educação, desenvolvimento profissional, normas, regulação e condições de emprego para enfermeiros; Todos os países possuindo planos para desenvolver enfermagem e obstetrícia; Maior investimento em todos os aspec- 
tos da enfermagem e obstetrícia; Mais enfermeiros, melhores treinamentos e empregos, com claro progresso na eliminação do déficit global de 9 milhões de enfermeiras e parteiras até 2030. Maior e melhor disseminação de práticas eficazes e inovadoras em enfermagem; Maior influência para enfermeiras e parteiras na política de saúde global e nacional, como parte de esforços mais amplos para garantir que as forças de trabalho da saúde estejam mais envolvidas na tomada de decisões $[\ldots]^{(3)}$.

A temática foi escolhida por apresentar relevância e avanços na área de enfermagem e de saúde como um todo, pois a campanha Nursing Now Brasil é recente e apresenta metas significativas para a profissão, apontando para o futuro mudanças de suma importância para os profissionais de enfermagem e principalmente para a população que necessita dos cuidados e assistência desses profissionais.

Diante dessas metas, e da indagação: "Qual o conhecimento e perspectivas dos enfermeiros de uma Unidade de Pronto Atendimento sobre a Campanha Nursing Now Brasil em seu cotidiano?", o objetivo desse estudo foi compreender os conhecimentos e perspectivas dos enfermeiros sobre a campanha Nursing Now Brasil.

\section{MÉTODO}

Trata-se de uma pesquisa descritiva exploratória, usando a metodologia "Bola de Neve", a ser desenvolvida em uma cidade do Noroeste do Paraná.

A pesquisa qualitativa permite o estudo de momentos privilegiados e particulares, dos quais emergem o sentido de um fenômeno social, permite considerar os diferentes aspectos de um fato particular e relacioná-lo ao contexto geral, além da capacidade de formular proposições ligadas à ação e à prática ${ }^{(4)}$.

Para identificação dos sujeitos foi utilizado a metodologia "Bola de Neve" ou "Cadeia de Informantes" que corresponde em uma metodologia de amostra não probabilística empregada em estudos sociais onde os indivíduos selecionados inicialmente indicam outros participantes que por sua vez sugerem novos sujeitos e assim sucessivamente, até que seja alcançado o ponto de saturação. Este ponto é atingido quando os recém-entrevistados passam a reproduzir os mesmos conteúdos já obtidos nas entrevistas anteriores, sem acrescentar novidades de dados à pesquisa $^{(5-6)}$.

Ao discutir o propósito de estratégias de "recrutamento de rede" Albuquerque discorre o método proposto Goodman em 1961, onde segundo a autora, os primeiros participantes contatados para a entrevista são as "sementes", estes devem ter ciência da sua localidade, do fato abordado, das pessoas que vivem na sua comunidade e estar qualificado sobre o assunto. Esse mesmo indivíduo (a "semente") apontará outra(s) pessoa(s) de seu convívio (ou de seu conhecimento) para que também participe $(m)$ da pesquisa, esses são os "filhos" das "sementes". Para que se tenha uma amostra considerável, deve-se selecionar um número inicial de pessoas (de "sementes"), que, preferencialmente, devem desempenhar certa liderança no campo a ser estudado e conhecer muitos membros na sociedade ${ }^{(7)}$.

Assim, para identificação dos sujeitos na amostra do estudo foi realizado primeiramente um convite aos enfermeiros da Unidade de Pronto Atendimento (UPA24h) de Sarandi/PR, estes foram as "sementes" do estudo, após autorização da pesquisa, foi agendado uma visita para a entrevista com as duas primeiras "sementes", estes ao térmico dos questionamentos indicaram duas "sementinhas". Para a identificação da amostra foi considerado ainda: ser enfermeiro(a); estar cadastrado no Conselho Regional de Enfermagem; e, ter trabalhado pelo menos dois anos no estabelecimento.

A coleta de dados ocorreu nos meses de março, abril e maio de 2020. Os participantes foram informados sobre os objetivos do estudo, e os procedimentos que serão adotados, sobre a livre opção em participar ou não, a possibilidade de negar a autorização retirando-se do estudo a qualquer momento que desejar, inclusive se já tiver iniciado a entrevista, e sobre o compromisso em garantir a livre participação e o anonimato das informações. Após o esclarecimento de dúvidas, os que concordaram em participar do estudo, assinaram o Termo de Consentimento Livre e Esclarecido (TCLE).

As entrevistas, foram gravadas para garantir maior interação e fidedignidade das respostas, e posteriormente foram transcritas de forma integral para análise dos dados.

Para percurso metodológico no tratamento dos dados foi utilizada a análise de conteúdo de Bardin, que emprega a manipulação de mensagens, comunicações, para confirmar os indicadores que permitam inferir sobre outra realidade que não a da mensagem. É o desvelar crítico.8 Por fim, a discussão foi sustentada com base no material científico disponível na área da saúde e afins, com base nos objetivos propostos.

A fim de preservar a identidade dos participantes os mesmos foram nomeados como heróis mundialmente conhecidos: Mulher Maravilha, Homem Aranha, Batman, Viúva Negra, Hulk, Mulher Invisível, Superman, Homem de Ferro, Flash e Capitão América, pois no período da coleta de dados deste estudo os profissionais de saúde passaram a ser reconhecidos popularmente como "heróis" ou "heróis de jaleco", por conta do importante papel que estavam desempenhando no combate ao novo Coronavírus (COVID-19), representados na figura 1.

O estudo foi desenvolvido em consonância com as diretrizes disciplinadas pela Resolução n. 466/12 do Conselho Nacional de Saúde, onde sofreu apreciação do Comitê Permanente de Ética em Pesquisa com Seres Humanos da Universidade Estadual de Maringá (COPEP) e foi aprovado pelo parecer 3.922.201. 


\section{RESULTADOS E DISCUSSÃO}

O estudo foi desenvolvido a partir da participação de 11 profissionais de enfermagem, exclusivamente enfermeiros, com total predominância da população feminina, atuantes especificamente no setor público como líderes da equipe de enfermagem.

Após análise de conteúdo foi possível identificar que os enfermeiros possuem diferentes percepções e conhecimentos frente a campanha Nursing Now Brasil, com isso foi realizado a construção de 3 categorias temáticas: "Diferentes percepções e conhecimentos da campanha Nursing Now Brasil pelos enfermeiros", "Desconhecimento dos enfermeiros sobre a campanha Nursing Now Brasil" e "Melhorias na enfermagem: esperança versus desvalorização".

Diferentes percepções e conhecimentos da campanha NursingNow Brasil pelos enfermeiros

Nesta categoria fica notória a relação de proximidade das diferentes percepções dos participantes, sendo possível identificar nos relatos que os mesmos acreditam que a campanha possui objetivos positivos para a enfermagem e destacam que a valorização da classe profissional ocorrerá e será desencadeada por meio da campanha NursingNow Brasil.

"Eu entendi que até que enfim alguém olhou para a enfermagem, a campanha vem para valorizar a enfermagem, mostrar o seu real papel, mostrar que o enfermeiro ele não está ali para ser um auxiliar e que ele tem uma função muito importante na saúde, aliás é o enfermeiro que faz a saúde acontecer". (Mulher Maravilha)

"A campanha ela vem para valorizar o profissional de enfermagem, essa valorização não é só do enfermeiro, é uma valorização que vai desde o técnico de enfermagem até o enfermeiro, é uma valorização do conhecimento científico". (Viúva Negra)

"[...] Deve ser uma campanha boa".(Batman)

"Essa campanha vem para valorizar nós enquanto profissionais e como profissão [...]".(Viúva Negra)

A história da enfermagem é ligada diretamente à desvalorização, visto que seu trabalho teve início por meio de mulheres e pessoas consideradas com baixo valor de estigma pela sociedade, como bêbados e prostitutas, desencadeando a submissão e a invisibilidade da profissão ${ }^{(10)}$.

Observou-se no estudo a presença da falta de valorização ao profissional de enfermagem nos dias atuais, pois, com base nos relatos os profissionais almejam meIhorias e valorização por meio da campa-

Figura 1: Representação da metologia Bola de Neve utilizada no estudo

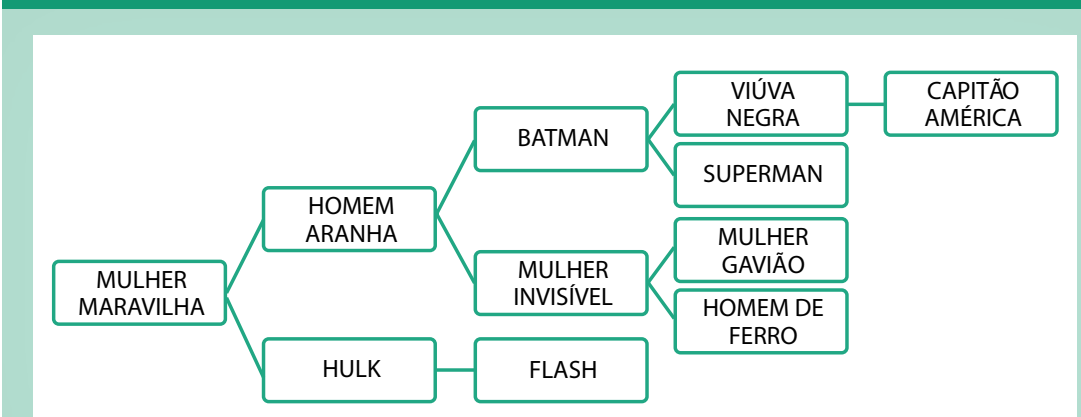

Fonte: Dados da Pesquisa, 2020

nha, este profissional realiza um papel fundamental na saúde e para isso deve estar motivado. A desvalorização ao profissional de enfermagem gera diversas implicações que afetam o desenvolvimento do processo do cuidar, causando falta de empenho ao exercício profissional, frustrações, sentimento de tristeza e revolta, gerando assim um atendimento de má qualidade ${ }^{(11)}$.

Desta forma,os autores adjuntos com os dados do estudo, inferem que os profissionais atuantes nos diferentes serviços de saúde devem sempre buscar a valorização da sua classe profissional, por meio da divulgação de seu trabalho, aplicação do conhecimento científico baseado em evidências no desenvolvimento da prática operacional e realizar o aprofundamento de campanhas e estudos que priorizam o desenvolvimento da enfermagem.

Desconhecimento dos enfermeiros sobre a campanha NursingNow Brasil

Apesar de relatos de conhecimento da campanha na categoria descrita acima, no decorrer do estudo foi possível evidenciar e identificar claramente a forte presença do desconhecimento por parte dos profissionais sobre a campanha NursingNow Brasil. Através das falas os entrevistados demonstram o desuso e a inaplicabilidade no ambiente de trabalho dos instrumentos e metas que a campanha oferece para toda a classe.

"Eu não sei realmente o que é e qual é o objetivo, eu ouvi falar no site do COREN (Conselho Regional de Enfermagem) e nos sites de enfermagem só que eu realmente não me atentei a ler e me aprofundar do assunto [...]". (Homem Aranha)

"Não tinha conhecimento desta campanha, nunca ouvi falar, deve ser uma campanha boa né, para avaliar o Enfermeiro ou para melhorar alguma coisa na área da assistência, eu imagino que seja isso [...]"..(Batman) 
"Eu não conheço ainda, nunca ouvi falar, pelo que eu estou percebendo se refere a enfermagem na atualidade, acho que é uma coisa boa [...]". (Hulk)

"[...] nunca tinha ouvido falar, deve ser algo positivo, mas eu desconheço.(Mulher Invisível)

“[...] desconheço essa campanha, eu acredito que seja algo para a profissão e de imediato né porque é "now" agora".(Superman)

"É eu não ouvi falar sobre essa campanha, acredito que seja algum movimento, algum chamamento da enfermagem, alguma coisa nesse sentido para mobilizar a categoria". (Homem de Ferro)

"Não conheço a campanha e não sei qual é o seu objetivo". (Flash)

"Eu tenho rede social e nunca vi nada, ruim não deve ser $\mathrm{NE}$ [...]".(Capitão América)

A campanha possui uma divulgação global em parceria com a Organização Mundial da Saúde (OMS) e o Conselho Internacional de Enfermeiros (CIN), com três anos de duração (2018-2020). Desde seu início a campanha foi divulgada em sites educacionais de enfermagem, revistas científicas e até mesmo foi discutida em debates e congressos, houve também o apoio de líderes e influentes políticos como a duquesa de Cambridge ${ }^{(12)}$.

Em decorrência da desvalorização adjunto com a baixa remuneração, a grande maioria os profissionais de enfermagem são obrigados a realizar uma dupla jornada de trabalho, que acarreta no desenvolvimento de danos negativos ao profissional, como o cansaço físico e mental e o desenvolvimento de doenças ocupacionais. Como consequência de uma jornada extensa e cansativa o profissional não possui tempo hábil para realizar leituras e atualizações profissionais que envolvam a sua classe profissional ${ }^{(13)}$.

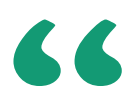

\section{Em decorrência da desvalorização} adjunto com a baixa remuneração, a grande maioria os profissionais de enfermagem são obrigados a realizar uma dupla jornada de trabalho, que acarreta no desenvolvimento de danos negativos ao profissional, como o cansaço físico e mental e o desenvolvimento de doenças ocupacionais.

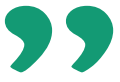

As instituições de saúde públicas e privadas devem ofertar aos profissionais atualizações e treinamentos que envolvam a equipe, para que assim não ocorra o desânimo e a mecanização do processo de trabalho, as atualizações devem ser ofertadas com o intuito de se realizar mudanças e melhorias durante o desenvolvimento da prática profissional.
Melhorias na enfermagem: Esperança versus Desvalorização

Muitas vezes a equipe de enfermagem se depara com diferentes situações, que assolam e dificultam o desenvolvimento do processo do cuidar fazendo com que os sentimentos dos profissionais sejam evidenciados. Dentro dos núcleos de significância foi possível identificar a presença da desvalorização da classe de enfermagem, porém, os profissionais se mostraram esperançosos com possíveis melhorias que possam vir a surgir com a divulgação e desenvolvimento da campanha.

"Qualquer melhoria é bem-vinda, por mínimo que seja é uma valorização para nós profissionais".(Mulher Maravilha)

"Tenho esperança que traga melhorias para a enfermagem[...]".(Mulher Invisível)

"Precisaria de uma maior divulgação dessa campanha para trazer melhorias, porque eu que trabalho e estou na linha de frente nunca tinha escutado, então eu acho que é uma campanha interessante só que não vai envolver boa parte dos profissionais". (Homem Aranha)

"A campanha já está trazendo melhorias para a enfermagem eu creio que nós vamos estar à frente de muita coisa e a profissão está muito bem avaliada".(Viúva Negra)

"Eu espero que traga melhorias para a nossa classe" (Hulk)

"Talvez ela possa trazer a valorização [...]". (Flash)

Mesmo diante do desconhecimento sobre as metas da campanha, os profissionais apresentam o sentimento de esperança, para que ocorra melhorias significativas em sua profissão. Com base 
Figura 2: Nuvem de palavras representando visualmente as palavras mais utilizadas no estudo pelos participantes

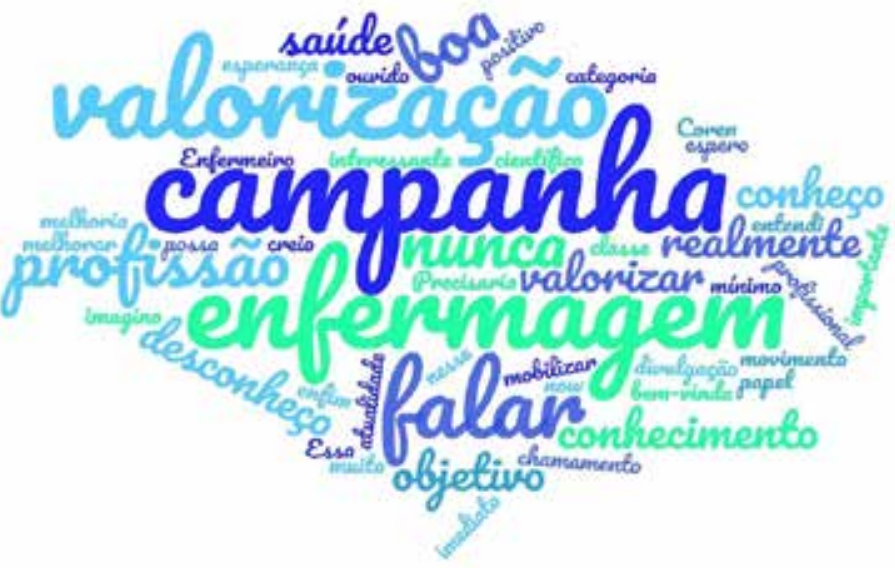

Fonte: Dados da Pesquisa, 2020

nas metas divulgadas pela campanha NursingNow Brasil o foco será pautado no desenvolvimento da educação, autonomia do profissional de enfermagem e formação de novos profissionais, garantindo assim um maior avanço científico da enfermagem, condições dignas de trabalho, avanços de práticas inovadoras e o crescimento da enfermagem baseada em evidências, resultando em um favorecimento do processo do cuidar ${ }^{(14)}$.

Diante aos resultados obtidos, desenvolvemos uma Nuvem de palavras com as principais nuances apresentadas pelos enfermeiros que fizeram parte desse estudo, destacando a enfermagem, a campanha e a valorização dessa profissão, que para muitos são verdadeiros heróis (Figura 2).

\section{CONCLUSÃO}

Por meio da realização deste estudo foi possível evidenciar e compreender o conhecimento dos profissionais enfermeiros diante da campanha Nursing Now Brasil e suas metas. Conclui-se que os enfermeiros possuem pouco conhecimento em relação a campanha e suas metas, demonstrando assim a não aplicabilidade no ambiente de trabalho e a falta de atualizações profissionais.

O estudo demonstrou também a presença da falta de valorização ao profissional de enfermagem, este profissional exerce um papel muito importante na saúde, e em decorrência da desvalorização ocorre o aparecimento de danos negativos, estes danos não afetam somente ao profissional e sim implicam no desenvolvimento do seu trabalho.

Sugere-se a divulgação mais ampla da Campanha, explorando mais as redes sociais e impulsionamento das mesmas pelos órgãos responsáveis, a fim de proporcionar mais informações sobre as metas da Nursing Now aos enfermeiros.

\section{Referências}

1. Cassiani SHB; Neto JCGL. Perspectivas da Enfermagem e a Campanha NursingNow. Revi Bras de Enf. 2018 Out.

2. OPAS. Pan American Health Organization. Expanding the role of nurses in Primary Health Care [Internet]. Washington, DC: PAHO. 2018 [cited $2018 \mathrm{Jul}$ 07]. Available from: http://iris.paho.org/xmlui/bitstream/handle/123456789/34958/9789275120033_eng.pdf? sequence $=6 \&$ isAllowed=y3. OLIVEIRA, J. E. P.; MONTENEGRO JUNIOR, M. M.; VENCIO, S. Diretrizes da Sociedade Brasileira de Diabetes 2017-2018. São Paulo: Editora Clannad, 2017.

3. COFEN. Conselho Federal de Enfermagem. NursingNow Brasil. 2019. Disponível em: http://nursingnowbrasil.com.br/\#quem-somos

4. Minayo MCS. Análise qualitativa: teoria, passos e fidedignidade. Ciênc Saud Colet. 2012.

5. Biernacki P; Waldorf D. Snowball Sampling: Problems and techniques of Chain Referral Sampling. SociologicalMethods\&Research, vol. $n^{\circ} 2$, November. 141-163p, 1981.

6. Albuquerque EM. Avaliação da técnica de amostragem "Respondent-drivenSampling" na estimação de prevalências de Doenças Transmissíveis em populações organizadas em redes complexas [dissertação]. Rio de Janeiro: Escola Nacional de Saúde Pública Sérgio Arouca - ENSP Ministério da Saúde
- Fiocruz; 2009.

7. Albuquerque EM. Avaliação da técnica de amostragem "Respondent-drivenSampling" na estimação de prevalências de Doenças Transmissíveis em populações organizadas em redes complexas [dissertação]. Rio de Janeiro: Escola Nacional de Saúde Pública Sérgio Arouca - ENSP Ministério da Saúde - Fiocruz; 2009.

8. Bardin L. Análise de conteúdo São Paulo: Edições 70, 2011, 229p

9. Brasil. Ministério da Saúde. Conselho Nacional de Saúde. Resolução 466/2012. Diretrizes e normas regulamentadoras de pesquisas envolvendo seres humanos. Brasília: Ministério da Saúde, 2012.

10. Gugel SCR, Duarte CS, Lima APL. Valorização da enfermagem brasileira: analisando aspectos históricos e de gênero. Revi Nurs. 2020 mar 23.

11. Lage CEB, Alves MS. (Des)valorização da enfermagem: implicações no cotidiano do enfermeiro. ReviCofen. 2016 dez 20.

12. Peate I. Global Nursing Now. British Journal of Nursing. 2019.

13. Bordignon M; Monteiro MI. Problemas de saúde entre profissionais de enfermagem e fatores relacionados. RevisEletTrim de Enferm. $2018 \mathrm{Jul}$. 14. COFEN. Conselho Federal de Enfermagem. NursingNow Brasil. 2019. Disponível em: http://nursingnowbrasil.com.br/\#quem-somos 